\title{
Vibration reduction of composite plates \\ by piezoelectric patches using a modified artificial bee colony algorithm
}

\section{Abstract}

In Current paper, power consumption and vertical displacement optimization of composite plates subject to a step load are carried out by piezoelectric patches using the modified multi-objective Elitist-Artificial Bee Colony (E-ABC) algorithm. The motivation behind this concept is to well balance the exploration and exploitation capability for attaining better convergence to the optimum. In order to reduce the calculation time, the elitist strategy is also used in Artificial Bee Colony algorithm. The voltages of patches, plate length/width ratios, ply angles, plate thickness/length ratios, number of layers and edge conditions are chosen as design variables. The formulation is based on the classical laminated plate theory (CLPT) and Hamilton's principle. The performance of the new ABC approach is compared with the PSO algorithm and shows the good efficiency of the new ABC approach. To check the validity, the transient responses of isotropic and orthotropic plates are compared with those available in the literature and show a good agreement.

\section{Keywords}

Smart composite plates, modified E-ABC algorithm.

\author{
Hadi Ghashochi-Bargh a \\ Mohammad Homayoune Sadr ${ }^{\text {* }}$ \\ ${ }^{a}$ Aerospace Engineering Department, \\ Center of Excellence in Computational \\ Aerospace Engineering, Amirkabir \\ University of Technology, Tehran, Iran \\ * Author email: Sadr@aut.ac.ir
}

\section{INTRODUCTION}

Composite materials are extensively used in various engineering applications such as aerospace structures, naval industry and other high-tech design, because of their superior performance. Thin laminated composite plates are structural components, which have considerable importance to build the lightweight structures. The use of piezoelectric sensor and actuator systems for the vibration suppression and shape control is fast becoming an essential tool in the design of smart thin plates. A piezoelectric actuator converts an electrical signal into a controlled physical displacement by producing a control 
force. If the control force is appropriate, the vibration of the structure may be suppressed adequately. In recent years piezoelectric materials and their use in structural dynamics have become a growing area of research. Devasia et al. (1993) used a simple numerical search algorithm to obtain optimal placement and sizing of the actuators for vibration suppression. Onoda and Hanawa (1993) used the genetic and simulating annealing methods to choose the optimal locations of the actuators in static shape control. Koconis et al. (1994) developed a solution scheme to find the optimal control voltages by minimizing an error function between the deformed shape and the desired shape. Varadan et al. (1997) studied optimal placement and size of piezoelectric actuators to reduce the radiation of sound into the space above a plate structure when excited by an acoustic pressure field produced by a noise source located below the plate. Han and Lee (1999) used genetic algorithms (GA) to find suitable locations of piezoelectric sensors and actuators of a cantilevered composite plate with considerations of controllability, observability and spillover prevention. Significant vibration reduction for the first three modes (controlled modes) has been achieved using the coupled positive position feedback in the vibration control experiment. Halim and Moheimani (2003) used spatial controllability and modal controllability to find the optimal placement of collocated actuator-sensor pairs for effective average vibration reduction over the entire structure. Sun and Tong (2005) described an energy optimization based method for finding the optimal control voltages that can actuate a structure shape close to the desired one within a given error. Qiu et al. (2007) investigated active vibration control of smart flexible cantilever plate by using discrete piezoelectric sensors and actuators. They presented a controller by combining PPF and proportional-derivative (PD) to suppress the first three vibration modes of the cantilever plate, including bending modes and torsional mode. Montazeri et al. (2008) utilized PSO to find the number, position and size of PZT sensors and actuators for active noise and vibration control of a simply supported laminated thin plate. They used the Hankel singular values of the state-space model of the system as the cost function to obtain the positions such that the closed-loop system is able to damp the maximum number of modes with an acceptable control effort and minimum complexity of the control system. Julai and Tokhi (2010) used genetic algorithm and particle swarm optimization for vibration suppression of flexible plate structures with all edges clamped. They designed the controllers based on direct optimization of the location of the detector and secondary source, and the controller parameters based on minimizing the MSE level of the error signal. Kapuria and Yasin (2013) examined the efficacy of directional actuation and sensing using piezoelectric fiber-reinforced composite (PFRC) actuators and sensors in active vibration suppression for smart fiber metal laminate (FML) plates.

In the present paper, vibration reductions of composite plates subject to a step load are carried out by piezoelectric patches using the modified multi-objective Elitist-Artificial Bee Colony (E-ABC) algorithm. In order to reduce the calculation time, the elitist strategy is used in Artificial Bee Colony algorithm. The voltages of patches, plate length/width ratios, ply angles, plate thickness/length ratios, number of layers and edge conditions are chosen as design variables. The performance of the E-ABC is compared with the PSO algorithm and shows the good efficiency of the E-ABC algorithm. 


\section{FORMULATION OF THE OPTIMIZATION PROBLEM}

Consider a thin composite laminated plate with dimensions of $\bar{a} \times \bar{b} \times h$ as shown in Fig. 1. Suppose that 4 piezoelectric actuators, each of dimensions $a \times b \times h_{P}$ are placed on the surface of the plate $\left(h_{P}=\right.$ thickness of actuators).

In this study, the plate is assumed to be thin so that the classical laminated plate theory (CLPT) assumptions are applied. Based on the CLPT, the displacement fields for a composite plate are given as (Jones, 1975; Ghashochi Bargh and Sadr, 2012):

$$
\begin{gathered}
\bar{u}(x, y, z, t)=u(x, y, t)-z \frac{\partial w(x, y, t)}{\partial x} \\
\bar{v}(x, y, z, t)=v(x, y, t)-z \frac{\partial w(x, y, t)}{\partial y} \\
\bar{w}(x, y, z, t)=w(x, y, t) .
\end{gathered}
$$

where $\bar{u}, \bar{v}$ and $\bar{w}$ are components of displacement at the arbitrary point, whilst $u, v$ and $w$ are corresponding ones on the middle surface $(\mathrm{z}=0)$.

According to Von Karman plate theory, components of strain field are obtained through the following equation:

$$
\bar{\varepsilon}=\left\{\begin{array}{l}
\bar{\varepsilon}_{x} \\
\bar{\varepsilon}_{y} \\
\bar{\gamma}_{x y}
\end{array}\right\}=\left\{\begin{array}{l}
\bar{u}_{, x} \\
\bar{v}_{, y} \\
\bar{u}_{, y}+\bar{v}_{, x}
\end{array}\right\}
$$

where $\bar{\varepsilon}$ is the in-plane strain vector.

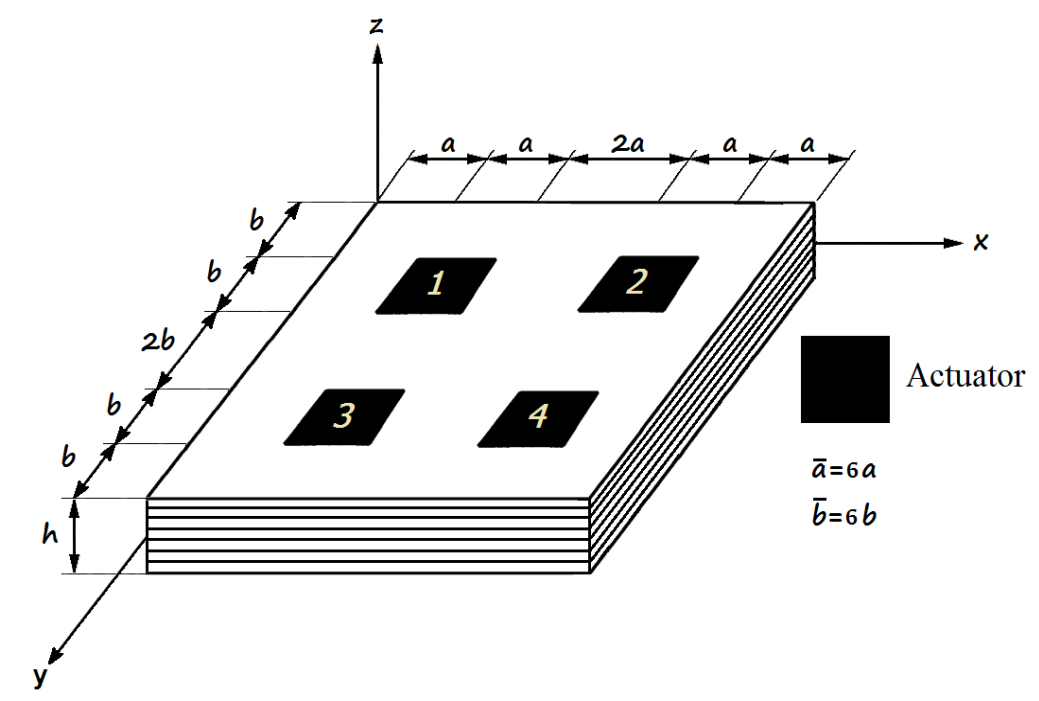

Figure 1: A laminated composite plate with distributed piezoelectric actuators. 
Substituting Eq. (1) into Eq. (2), the in-plane strain vector is expressed as:

$$
\bar{\varepsilon}=\varepsilon+z \psi=\left\{\begin{array}{l}
u_{, x} \\
v_{, y} \\
u_{, y}+v_{, x}
\end{array}\right\}+z\left\{\begin{array}{l}
-w_{, x x} \\
-w_{, y y} \\
-2 w_{, x y}
\end{array}\right\} .
$$

where $\varepsilon$ and $\psi$ are the in-plane strain vector at the mid-plane and the curvature strain vector, respectively.

Piezoelectric materials generate an electrical signal when subjected to mechanical strains and vice versa. These effects are known as direct and inverse piezoelectric effects and can be written in the form of two constitutive equations (Elshafei 1996):

$$
\begin{aligned}
& \{D\}=[e]^{T}\{\bar{\varepsilon}\}+[\in]\{E\}, \\
& \{\bar{\sigma}\}=[\bar{Q}]\{\bar{\varepsilon}\}-[e]\{E\} .
\end{aligned}
$$

where $\{D\}$ is the electric displacement, $[e]$ is the piezoelectric stress coefficient matrix, $[\in]$ is the permittivity matrix, $\{E\}$ is the electric field intensity, $\{\bar{\sigma}\}$ is the in-plane stress vector and $[\bar{Q}]$ is the planestress stiffness coefficients matrix.

The element has been assumed with one electrical degree of freedom at the top of the piezoelectric actuator patches. Electrical potential has been assumed to be constant over an element and vary linearly through the thickness of piezoelectric patch. We assume that the electric field vector $\{E\}$ can be defined by the electrical potential $\phi$ as:

$$
\{E\}=-\nabla \phi
$$

Hamilton's principle is used to determine the equation of motion of the plate and piezoelectric material system. The variational formulation for the structural system should be established. Hamilton's principle is written as:

$$
\int_{t 1}^{t 2} \delta(T-U) d t+\int_{t 1}^{t 2} \delta W d t=0
$$

where $\delta($.$) denotes the first variation, \mathrm{T}$ and $\mathrm{U}$ are the total kinetic energy and potential energy of the plate and piezoelectric material system, $\mathrm{W}$ is the work done by external loads, and $\mathrm{t} 1$ and $\mathrm{t} 2$ are the integration time limits.

The total kinetic energy of the plate and piezoelectric patch consists of the kinetic energies of the base plate and the piezoelectric layer. For thin plate, the general expression for the kinetic energy is: 


$$
T=\frac{1}{2} \rho h \iint\left\{\dot{u}^{2}+\dot{v}^{2}+\dot{w}^{2}\right\} d x d y=\frac{1}{2}\{\dot{d}\}^{T}\left[M^{e}\right]\{\dot{d}\}
$$

where $\left[M^{e}\right]$ is the element mass matrix and $\{\mathrm{d}\}$ is a column matrix which contains the element's degrees of freedom.

The total potential energy of the plate and piezoelectric patch system includes the strain energy of the base plate, and the strain energy and electric potential energy of the piezoelectric layer. The total potential energy can be written as:

$$
\begin{gathered}
U=\frac{1}{2} \iiint\left[\{\bar{\varepsilon}\}^{T}\{\bar{\sigma}\}-\{E\}^{T}\{D\}\right] d V \\
=\frac{1}{2} \iiint\left[\{\bar{\varepsilon}\}^{T}[\bar{Q}]\{\bar{\varepsilon}\}-\{\bar{\varepsilon}\}^{T}[e]\{E\}-\{E\}^{T}[e]^{T}\{\bar{\varepsilon}\}-\{E\}^{T}[\in]\{E\}\right] d V .
\end{gathered}
$$

where

$$
\begin{gathered}
\frac{1}{2} \iiint\{\bar{\varepsilon}\}^{T}[\bar{Q}]\{\bar{\varepsilon}\} d V=\frac{1}{2} \iint\left[\{\varepsilon\}^{T}[A]\{\varepsilon\}-2\{\varepsilon\}^{T}[B]\{\psi\}+\{\psi\}^{T}[D]\{\psi\}\right] d x d y \\
=\frac{1}{2}\{d\}^{T}\left[K_{u u}^{e}\right]\{d\} .
\end{gathered}
$$

In the equation above, $[\mathrm{A}],[\mathrm{B}]$ and $[\mathrm{D}]$ are the matrices of stiffness coefficients and $\left[K_{u u}^{e}\right]$ is the element elastic stiffness matrix.

The work done by the applied forces can be expressed in the form:

$$
W=\iint\{d\}^{T}\{f\} d x d y
$$

where $f$ is the column matrix of forces at reference lines, which corresponds to d. Of course, the only non-zero entries in $\mathrm{f}$ correspond to $\mathrm{w}$ values in $\mathrm{d}$ in view of the fact that the loading acts in the $\mathrm{z}$ direction.

Substituting equations (7)-(9) into equation (6) and using equations (3)-(5) the dynamic matrix equation can be written as (Chandrashekhara and Agarwal, 1993; Correia et al., 2000):

$$
\left[M^{e}\right]\{\ddot{d}\}+\left[K_{u u}^{e}\right]\{d\}=\left\{F^{e}\right\}-\left\{F_{v}^{e}\right\} ;\left\{F_{v}^{e}\right\}=\left[K_{u \phi}^{e}\right]\left\{\phi_{0}^{e}\right\}
$$

where $\left\{F^{e}\right\}$ is the external mechanical force vector, $\left\{F_{v}^{e}\right\}$ is a additional force vector due to the voltage $\phi_{0}^{e}$ applied to the actuators and $\left[K_{u \phi}^{e}\right]$ is the coupling matrix between elastic mechanical and electrical effects.

For the whole structure, assembling the element equations gives the global dynamic equation:

$$
[M]\{\ddot{\bar{d}}\}+[K]\{\bar{d}\}=\{F\}+\left\{F_{v}\right\}
$$


where $[K]$ and $[M]$ are the square symmetric, positive-definite structure stiffness and consistent mass matrices and $\{\bar{d}\}$ is a vector, which includes the degrees of freedom for the whole structure.

To obtain transient response of the plate for the total time, Newmark's direct integration technique is used. This technique involves parameters $\gamma$ and $\delta$ that control the accuracy and stability of the technique. The choice of $\gamma=0.5$ and $\delta=0.25$ corresponds to a constant average-acceleration method, which is known to give an unconditionally stable algorithm in linear problems.

Non-dimensional vertical displacements of laminated plates are defined as:

$$
\bar{w}^{\prime}=100 \bar{w}\left(\frac{E_{2} h^{3}}{q_{0} \bar{a}^{4}}\right) .
$$

In this paper, our goal is to seek the voltage distribution of piezoelectric actuators that minimize the maximum vertical displacement of composite plate $\left(\bar{w}^{\prime \text { maximum }}\right)$ and energy consumption.

The energy consumed by the piezoelectric actuators is abstractly defined as (Sun and Tong, 2003; Kang and Tong, 2008):

$$
E_{k}=z_{0} V_{k}^{T} V_{k} \quad, \mathrm{k}=1 \ldots 4
$$

where $\mathrm{V}_{\mathrm{k}}$ is the voltage of piezoelectric patches and $\mathrm{z}_{0}$ is a weighting coefficient representing electric conductance for the actuators $\left(\mathrm{z}_{0}=1\right)$.

The optimal design problem can be stated as follows:

$$
\begin{array}{ll}
\text { Find } & \mathrm{V}=\left(\mathrm{V}_{1}, \mathrm{~V}_{2}, \mathrm{~V}_{3}, \mathrm{~V}_{4}\right), \\
\text { Minimize } & \bar{w}^{\prime \text { maximum }} \text { and } \sum_{k=1}^{4} E_{k}, \\
\text { Subject to } & -400 \leq \mathrm{V}_{\mathrm{k}} \leq 400 \quad, \mathrm{k}=1 \ldots 4
\end{array}
$$

The optimal voltages are searched with the modified E-ABC algorithm.

In the multi-objective optimization of laminated composite plates, the objective functions combined with each other through the weighted summation method. The obtained single objective function is then optimized using modified E-ABC algorithm. To simultaneously minimize the maximum vertical displacement of plate and energy consumption, the objective function is considered in the form $\mathrm{f}(\mathrm{V})$ as a function of voltage of piezoelectric patches which is defined as (Sadr and Ghashochi Bargh, 2012):

$$
f_{i}(V)=W_{1} \frac{\left(w^{\prime \max \text { imum }}\right)_{i}}{\sum_{i=1}^{s}\left(w^{\prime \max \text { imum }}\right)_{i}}+W_{2} \frac{\left(\sum_{k=1}^{4} E_{k}\right)_{i}}{\sum_{i=1}^{s}\left(\sum_{k=1}^{4} E_{k}\right)_{i}}
$$

where $\mathrm{W}_{1}$ and $\mathrm{W}_{2}$ are the weighting coefficients summing the two objective functions to have a single fitness function and $\mathrm{s}$ is number of the best memorized solutions. In this paper, the optimization results are given for $\mathrm{W}_{1}=0.5$ and $\mathrm{W}_{2}=0.5$. 
As seen, the roulette wheel scheme is employed in the objective function, in which each solutions $\left(\bar{w}^{\prime \text { maximum }}\right.$ and energy consumption) is assigned a value within the range $[0,1]$.

\section{MODIFIED ELITIST-ARTIFICIAL BEE COLONY ALGORITHM}

The ABC algorithm is one of the most recently defined algorithms by Dervis Karaboga (2005), motivated by the intelligent behavior of honey bees. In the ABC algorithm, the colony of artificial bees contains three groups of bees: employed bees, onlookers and scouts. A bee waiting on the dance area for making decision to choose a food source is called an onlooker and a bee going to the food source visited by itself previously is named an employed bee. A bee carrying out random search is called a scout. In the $\mathrm{ABC}$ algorithm, first half of the colony consists of employed artificial bees and the second half constitutes the onlookers. For every food source, there is only one employed bee. In other words, the number of employed bees is equal to the number of food sources around the hive. The employed bee whose food source is exhausted by the employed and onlooker bees becomes a scout.

In this study, $\mathrm{ABC}$ algorithm is used as the optimization method and to improve the quality of the solution, the elitist strategy is used in the algorithm. In this strategy, the onlooker bee operator generates a more diverse set of solutions near the best solutions so far until the end of each cycle in the algorithm. From this modification on the onlooker bee, the term Elitist is added to the name of this version of the ABC. A parameter is defined to control the amount of generation of solutions near the best solutions. In the algorithm, this parameter is assumed $20 \%$ of the best solutions. Thus, the same number of solutions will always be generated from a solution. As the value of parameter increases, causing the $\mathrm{ABC}$ search to become more localized. Thus, using large value of parameter may prevent the ABC from finding the global optimum. A selection procedure based on the fitness function picks best food sources and replaces sources in algorithm. In addition, the E-ABC preserves only the best design in every cycle. The elitist strategy can be stated as follows (Mezura-Montes and Velez-Koeppel, 2010):

$$
v_{l}^{g}=x_{l}^{g}+\phi_{i j}\left(x_{l}^{g}-x_{l j}\right)
$$

where $j \in\{1,2, \ldots, D\}$ is random generating index, 1 is equal to the number of the best solutions depending on the value of defined parameter and $\mathrm{D}$ denotes the number of optimization parameters. $v_{l}^{g}, x_{l}^{g}$ and $\mathrm{x}_{\mathrm{lj}}$ are a set of new feasible solutions near the best solutions, the best solutions and the feasible solutions, respectively. $\phi_{i j}$ is random number between $[0,1]$

Also, the search form of $\mathrm{ABC}$ is good at exploration but poor at exploitation. Therefore, to improve the performances of $\mathrm{ABC}$, we also propose to make two other major changes by introducing the inertia weight and acceleration coefficient to modify the search process. The use of the inertia weight and acceleration coefficient provides a balance between global and local exploration and exploitation, and results in fewer iterations on average to find a sufficiently optimal solution.

The operation process can be expressed as the following form (Li et al. 2012):

$$
v_{i j}=w \cdot x_{i j}+2\left(\phi_{i j}-0.5\right)\left(x_{i j}-x_{k j}\right) \Phi \text {. }
$$


where $k \in\{1,2, \ldots, S N\}$ is random generating index, but $\mathrm{k}$ must be different from i and SN is the size of food sources. $\mathrm{v}_{\mathrm{ij}}$ is the new modified feasible solution that depending on its previous solution $\mathrm{x}_{\mathrm{ij}}$. $\mathrm{w}$ is the inertia weight which controls impacts of the previous solution $\mathrm{x}_{\mathrm{ij}} . \Phi$ is the acceleration coefficient that could control the maximum step size. However, if the global fitness is very large, bees are far away from the optimum values. So a big correction is needed to search the global optimum solution and then $\mathrm{w}$ and $\Phi$ should be bigger values. Conversely, only a small modification is needed, then $\mathrm{w}$ and $\Phi$ must be smaller values. So in order to further improve the search efficiency of the bees, w and $\Phi$ are decreased linearly from 0.9 to 0.4 during a run.
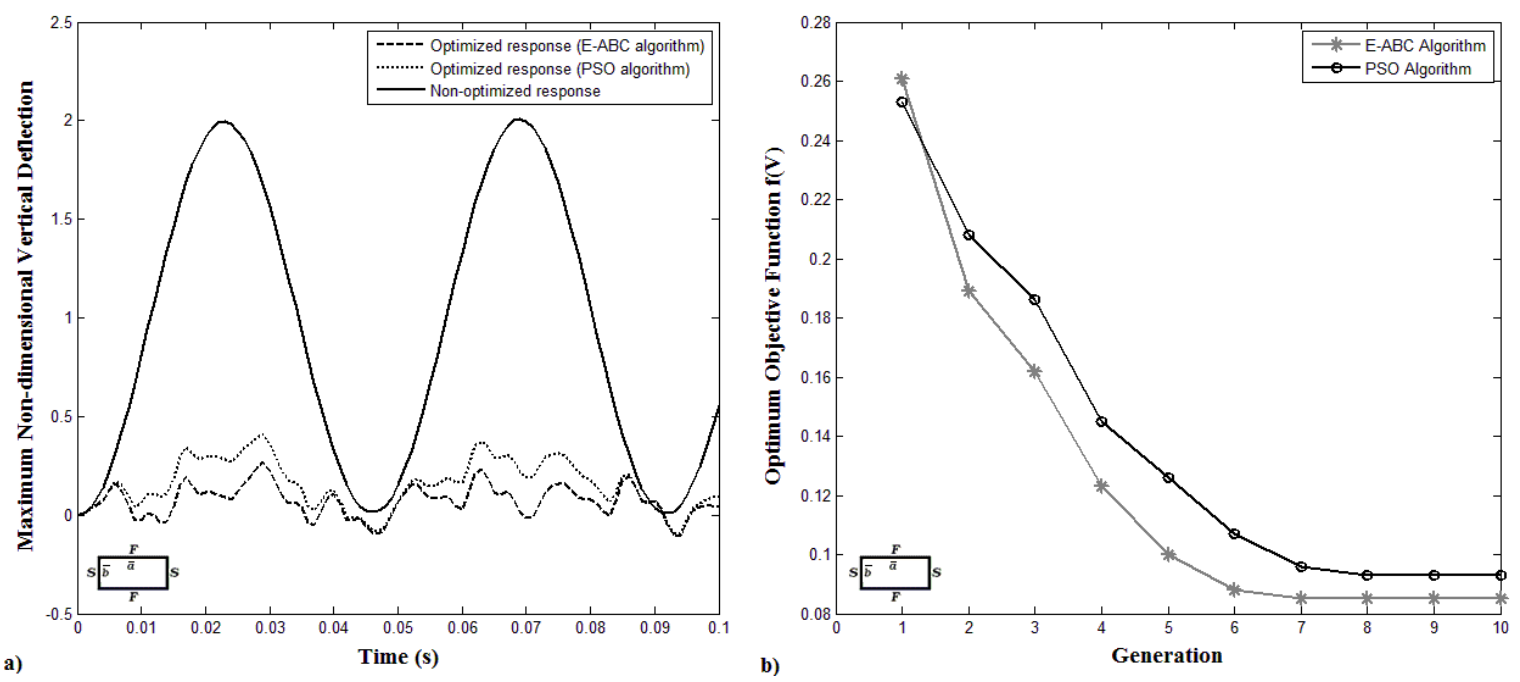

Figure 2: Comparison of the E-ABC and PSO results for SFSF edge condition for symmetrically 6-layered square composite plate with the same initial populations $(\bar{a} / \bar{b}=1, h / \bar{a}=0.005)$.

The convergence rate of objective function with the same number of generations for E-ABC and PSO (Rojas et al., 2004) for symmetrically 6-layered square composite plate with $[0 / 0 / 0]_{\mathrm{S}}$ stacking sequence is shown in Fig. 2(a) and (b). In this Case, the optimal energy consumption of piezoelectric patches obtained by E-ABC and PSO are 343162 and 414740, respectively. It is clear from Fig. 2(a) and (b) that, for the optimization problem considered, E-ABC converges at a faster rate (around 6 generations) compared to that for PSO (around 8 generations) with the initial population of 14 . In addition, it is concluded that using of E-ABC provides a much higher convergence and reduced the CPU time in comparison with the PSO.

\section{RESULTS AND DISCUSSIONS}

The maximum vertical displacement and energy consumption of composite laminates is minimized for different plate aspect ratios, ply angles, number of layers, boundary conditions and plate thickness/length ratios using the piezoelectric patches. 
The laminates are symmetric and made of AS/3501 graphite/epoxy material. The material properties of the laminas and piezoelectric patches are given as below (Vinson and Sierakowski, 1986; Ghashochi-Bargh and Sadr, 2013; Sun and Huang, 2000):

Composite layers: $\mathrm{E}_{1}=138 \mathrm{GPa}, \mathrm{E}_{2}=8.96 \mathrm{GPa}, \mathrm{G}_{12}=7.1 \mathrm{GPa}, \nu_{12}=0.3, \rho=1520 \mathrm{~kg} / \mathrm{m}^{3}$.

Piezoelectric patches: $\mathrm{E}_{1}=\mathrm{E}_{2}=20 \mathrm{GPa}, \mathrm{G}_{12}=0.775 \mathrm{GPa}, \mathrm{v}_{12}=0.29, \rho=1800 \mathrm{~kg} / \mathrm{m}^{3}$,

$\mathrm{e}_{31}=\mathrm{e}_{32}=0.046 \mathrm{C} / \mathrm{m}^{2}, \mathrm{e}_{31}=0 \mathrm{C} / \mathrm{m}^{2}, \in_{33}=0.1062 \times 10^{-9} \mathrm{~F} / \mathrm{m}$.

Each of the lamina is assumed to be same thickness $(h / \bar{a}=0.01$ or $h / \bar{a}=0.005)$.

The transient responses of isotropic and orthotropic plates are compared with the results of numerical values carried out by Chen and Dawe (1996) and Maleki et al. (2012), respectively.

Figure 3 shows center deflection of square isotropic panel $(100 \mathrm{~mm} \times 100 \mathrm{~mm} \times 1 \mathrm{~mm})$ under uniformly distributed step load of intensity $\mathrm{q}=0.0231 \mathrm{MPa}$ over the whole panel surface with all edges simply supported. It is seen that the present result has excellent agreement with the one obtained by Chen and Dawe. The material properties used in this example are:

$\mathrm{E}=205 \mathrm{GPa}, \mathrm{v}=0.3, \rho=7900 \mathrm{~kg} / \mathrm{m}^{3}$.

Figure 4 illustrates time histories of non-dimensional center deflection of laminated square plates $[30 /-30]$ s under conventional blast load with the clamped edges. It is seen that there is a good agreement between results of present approach and the published paper, for the transient responses of orthotropic panel. In this example, the following geometrical and material properties are used:

$\bar{a}=\bar{b}=1.27 \mathrm{~m}, h=0.0254 \mathrm{~m}, \rho=1610 \mathrm{~kg} / \mathrm{m}^{3}$,

$\mathrm{E}_{1}=131.69 \mathrm{GPa}, \mathrm{E}_{2}=8.55 \mathrm{GPa}, \mathrm{G}_{12}=6.67 \mathrm{GPa}, \mathrm{v}_{12}=0.3$.

The conventional blast loading is defined as:

$$
q(x, y, t)=q_{0}\left(1-\frac{t}{t_{2}}\right) e^{-\alpha_{1} t / t_{2}} .
$$

where $\mathrm{t}_{2}=0.004 \mathrm{~s}, \alpha_{1}=1.98$, and $\mathrm{q}_{0}=68.95 \mathrm{kPa}$.

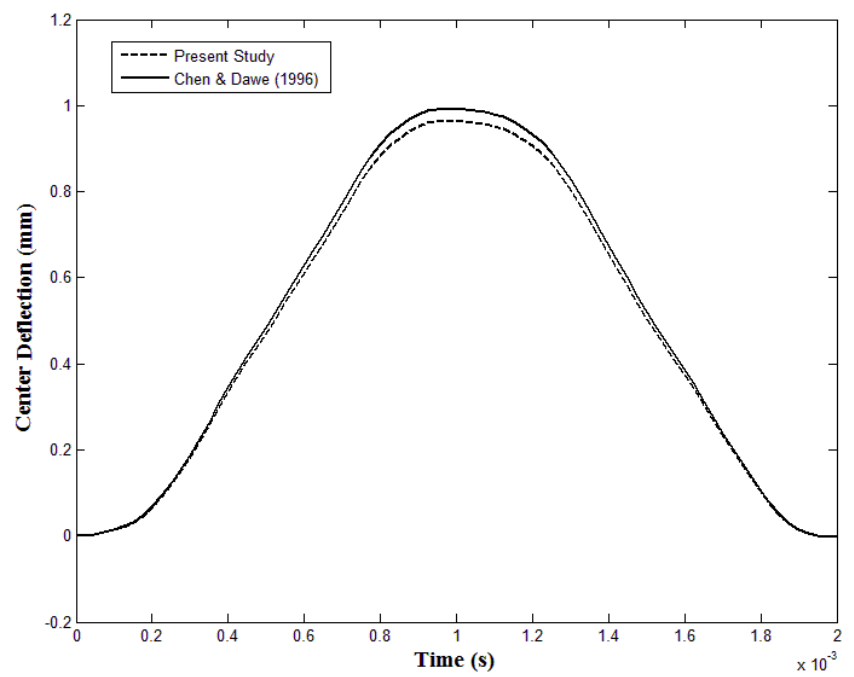

Figure 3: Response of isotropic, square, simply supported panel to uniformly distributed step load. 


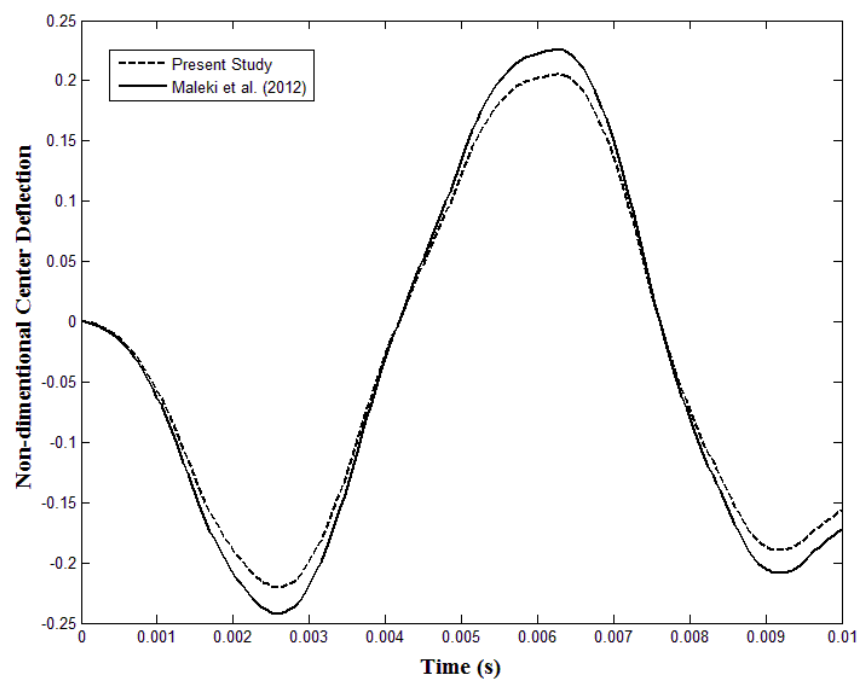

Figure 4: Response of orthotropic, square, clamped panel to conventional blast load.

The optimization results given in this paper are carried out for symmetrically composite plate $(100 \mathrm{~mm} \times 100 \mathrm{~mm})$ under uniformly distributed step load of intensity $\mathrm{q}=0.001 \mathrm{~Pa}$ over the whole plate surface with the different combinations of free (F), simply supported (S) and clamped (C) edge conditions. Consider the plate with known original shape and actuator configurations where the desired shape is specified (see Fig. 1). So the aim is to find the actuators voltages, which minimize the maximum vertical displacement of composite laminated plate by consuming minimum energy. The effects of the edge conditions, plate length/width ratios, stacking sequences and plate thickness/length ratios on the optimum design are shown for symmetric composite laminated plates in Fig. 5, 6, 7, 8 and 9. As inferred from the results, the good efficiency of the E-ABC search strategy and its ability to provide highquality solutions is confirmed for various solutions. 

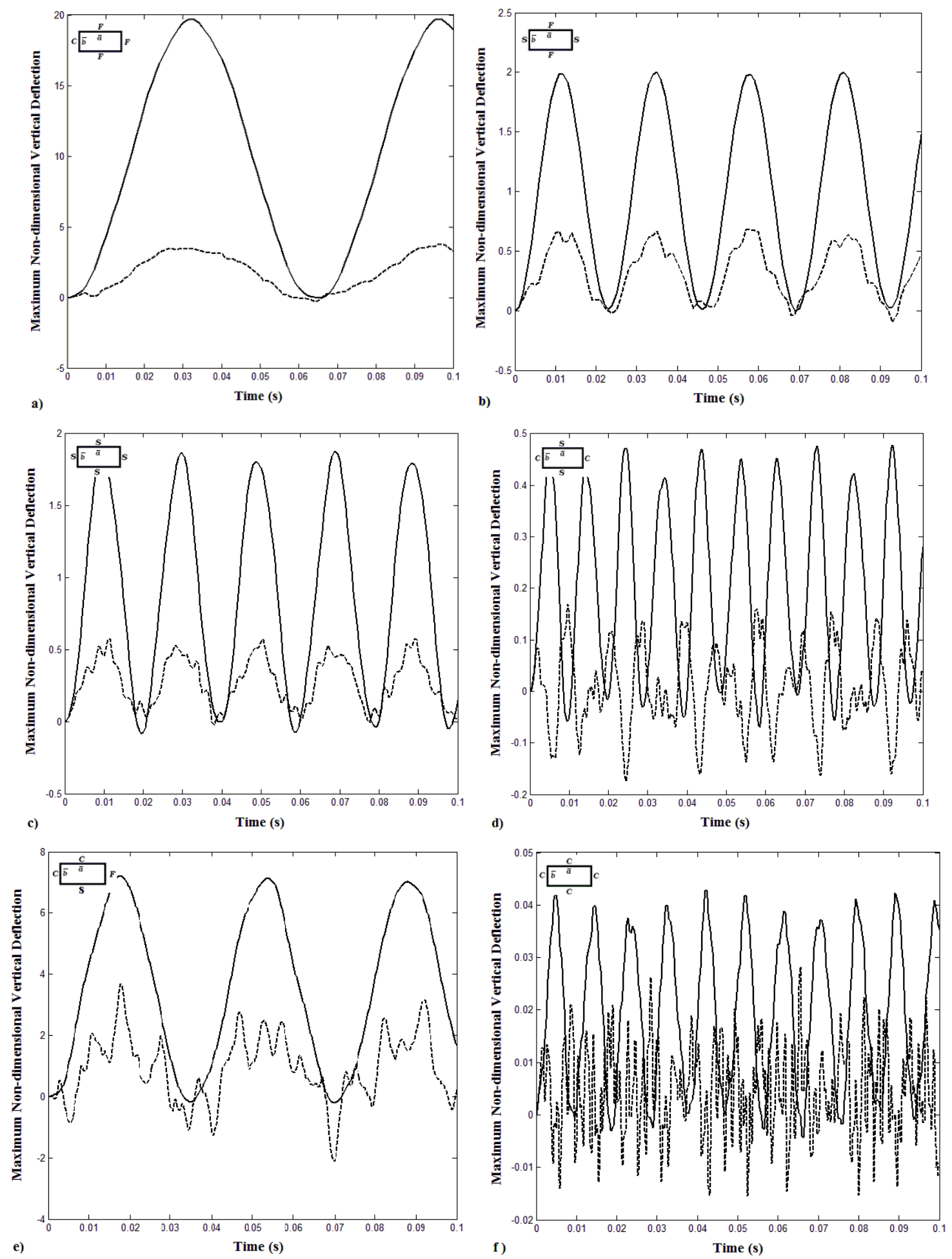

Figure 5: Optimum solutions of symmetric 6-layered plates obtained by E-ABC algorithm for different boundary conditions and $[0 / 0 / 0]_{S}$ stacking sequence $(\bar{a} / \bar{b}=1, h / \bar{a}=0.01)$ : (a) CFFF, (b) SFSF, (c) SSSS, (d) CSCS, (e) CCFS, (f) CCCC;-Non-optimized response, ---Optimized response. 

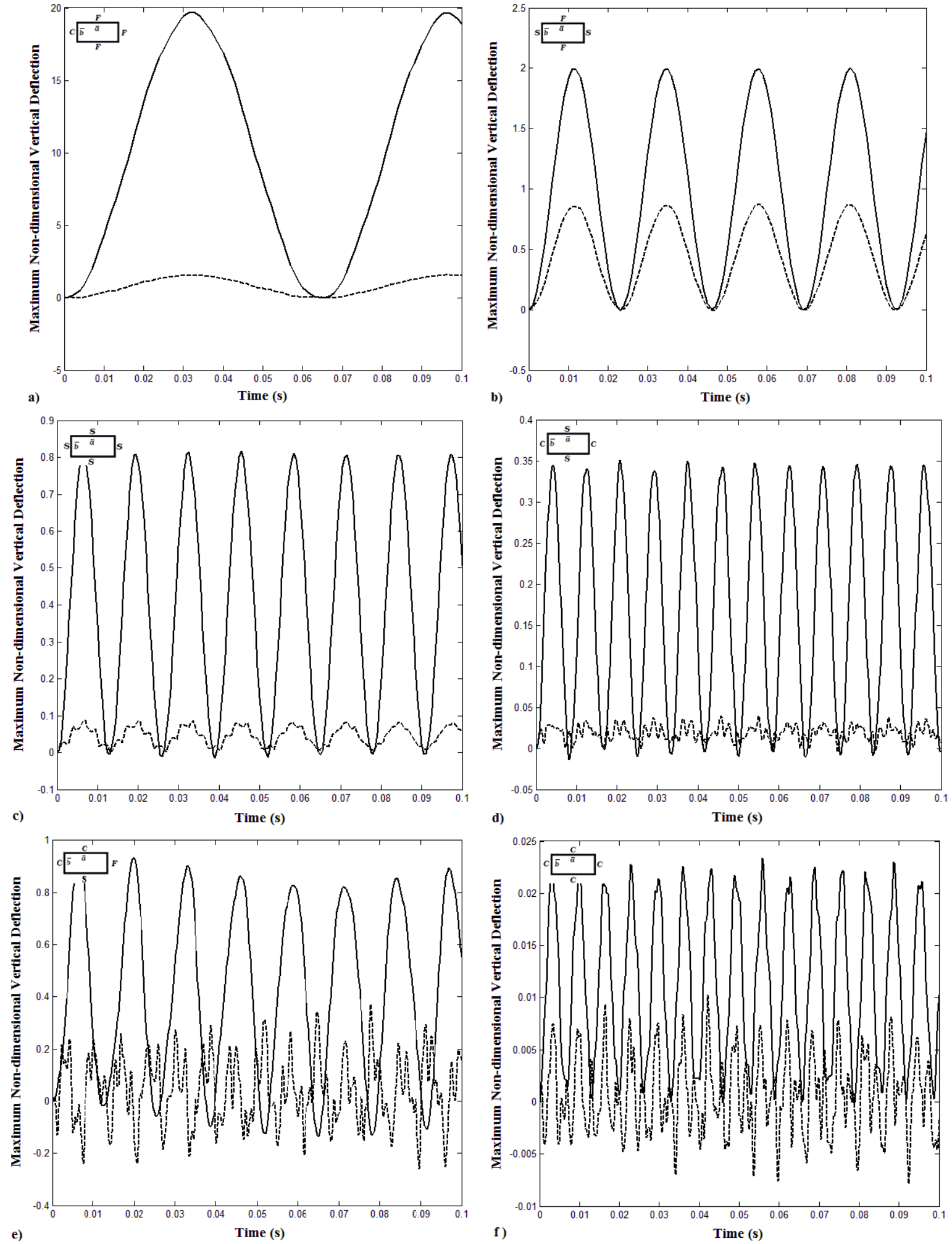

Figure 6: Optimum solutions of symmetric 6-layered plates obtained by E-ABC algorithm for different boundary conditions and $[0 / 0 / 0]_{S}$ stacking sequence $(\bar{a} / \bar{b}=2, h / \bar{a}=0.01):$ (a) CFFF, (b) SFSF, (c) SSSS, (d) CSCS, (e) CCFS, (f) CCCC;--Non-optimized response, ---Optimized response. 

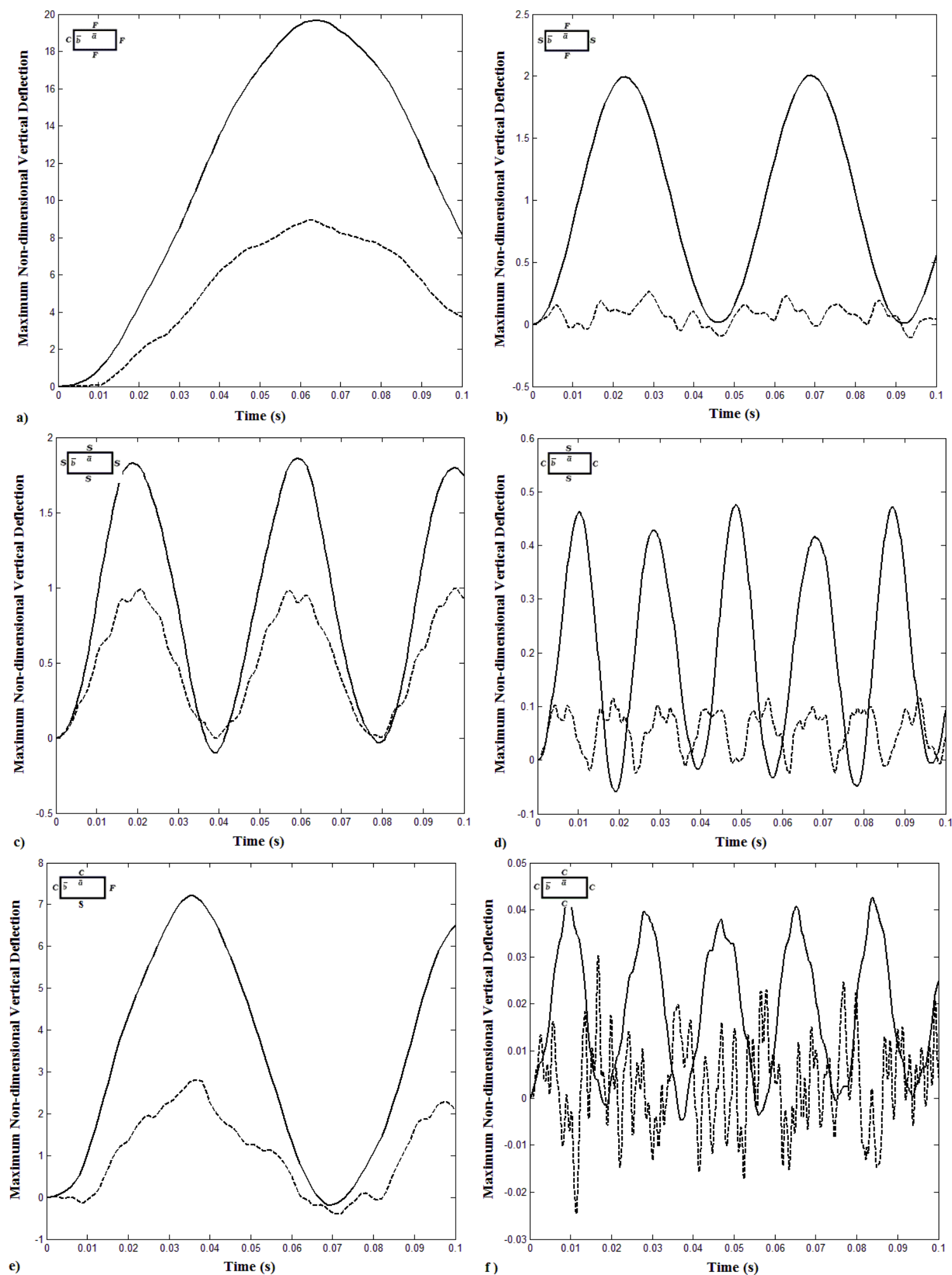

Figure 7: Optimum solutions of symmetric 6-layered plates obtained by E-ABC algorithm for different boundary conditions and $[0 / 0 / 0]_{S}$ stacking sequence $(\bar{a} / \bar{b}=1, h / \bar{a}=0.005):$ (a) CFFF, (b) SFSF, (c) SSSS, (d) CSCS, (e) CCFS, (f) CCCC;-Non-optimized response, ---Optimized response.

Latin American Journal of Solids and Structures 11 (2014) 1846-1863 

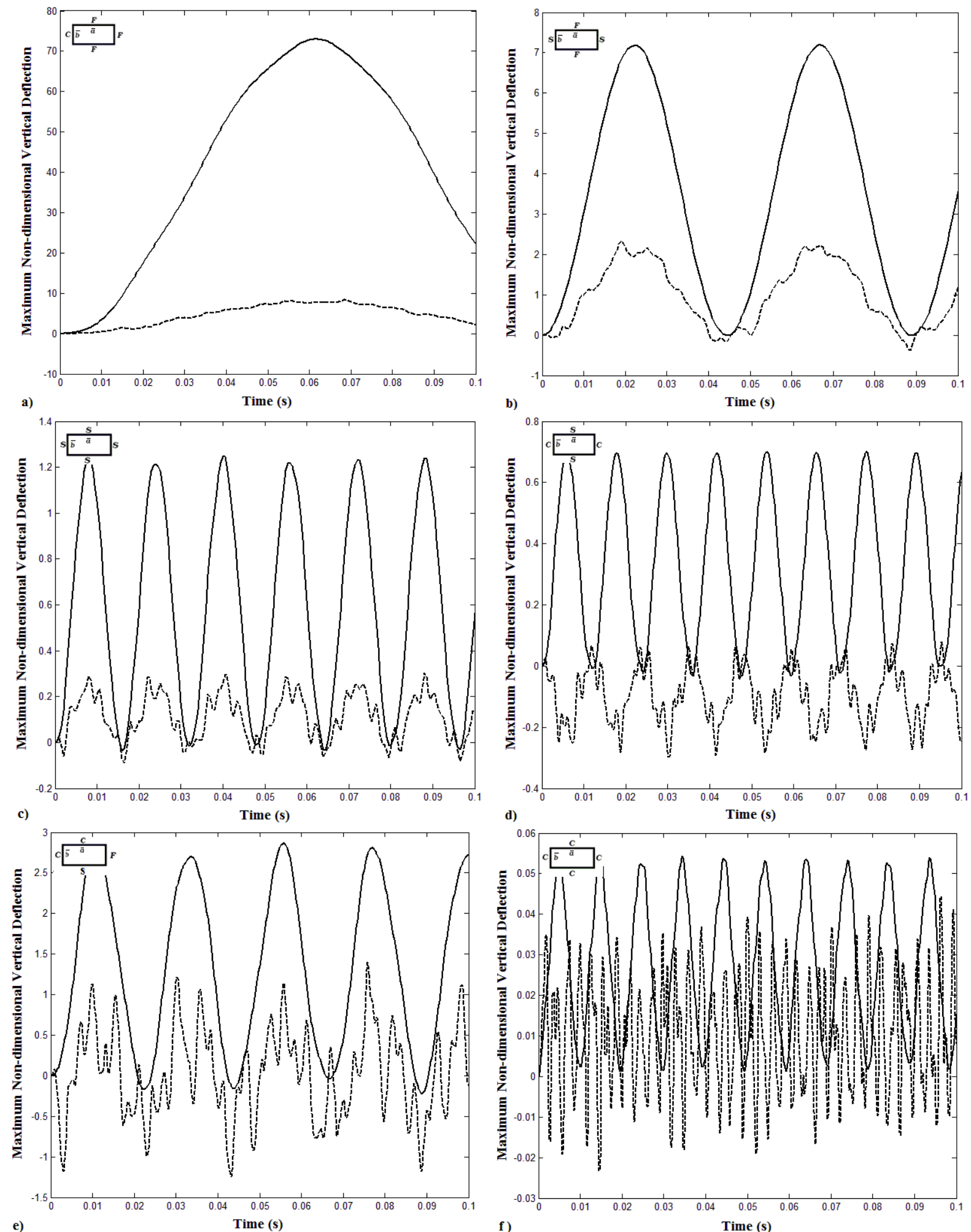

Figure 8: Optimum solutions of symmetric 6-layered plates obtained by E-ABC algorithm for different boundary conditions and $[45 /-45 / 45]_{S}$ stacking sequence $(\bar{a} / \bar{b}=1, h / \bar{a}=0.01):$ (a) CFFF, (b) SFSF, (c) SSSS, (d) CSCS, (e) CCFS, (f) CCCC;-Non-optimized response, ---Optimized response. 

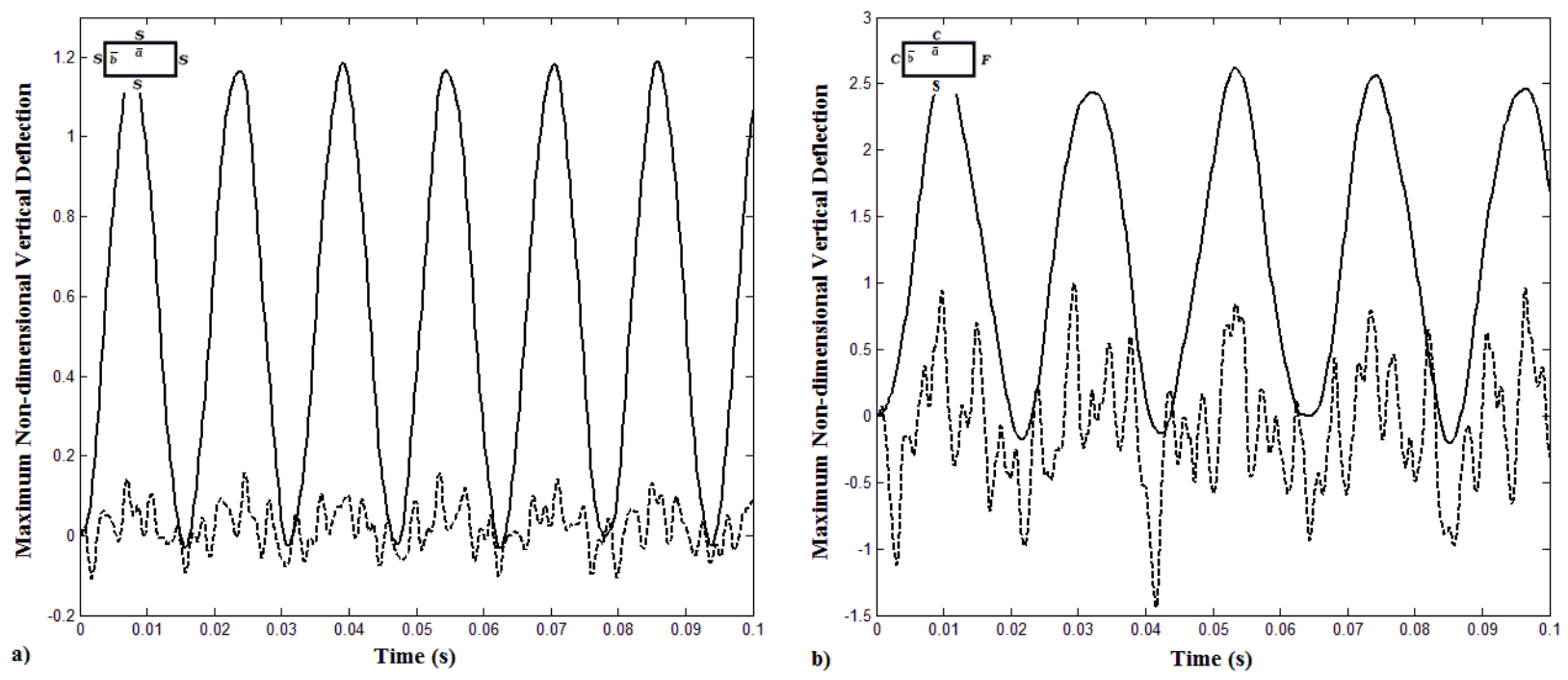

Figure 9: Optimum solutions of symmetric 10-layered plates obtained by E-ABC algorithm for different boundary conditions and $[45 /-45 / 45 /-45 / 45]_{\mathrm{S}}$ stacking sequence $(\bar{a} / \bar{b}=1, h / \bar{a}=0.01)$ : (a) SSSS, (d) CCFS;-Non-optimized response, --Optimized response.

Table 1, 2, 3 and 4 also represent the optimal voltages of the piezoelectric patches, which minimize the maximum vertical displacement of composite laminated plate by consuming minimum energy. In addition, the optimum voltages of patches are substantially influenced for edge conditions, plate thickness/length ratios and $\bar{a} / \bar{b}$ ratios. As seen, the optimum voltages of the piezoelectric patches are not influenced substantially and approach a limiting value by changing the stacking sequences and number of layers with the same plate thickness/length ratios. As inferred from Fig. 5 and 8, transient responses of composite plates are changed by changing the stacking sequences with the same optimum voltages of the piezoelectric patches. It is also clear from Table 1, 2 and 3 that the optimum voltages of patches 1 and 3 and optimum voltages of patches 2 and 4 approach a same value for symmetric boundary conditions.

\begin{tabular}{cccccc}
\hline \hline \multirow{2}{*}{ BCs } & $\bar{a} / \bar{b}$ & {$\left[\mathrm{~V}_{1} / \mathrm{V}_{2} / \mathrm{V}_{3} / \mathrm{V}_{4}\right]_{\mathrm{Opt}}$} & $\mathrm{BCs}$ & $\bar{a} / \bar{b}$ & {$\left[\mathrm{~V}_{1} / \mathrm{V}_{2} / \mathrm{V}_{3} / \mathrm{V}_{4}\right]_{\mathrm{Opt}}$} \\
\hline CFFF & 1 & {$[-158 /-51 /-156 /-52]$} & $\mathrm{CSCS}$ & 1 & {$[-155 /-21 /-158 /-22]$} \\
& 2 & {$[-45 /-291 /-45 /-291]$} & & 2 & {$[-190 /-301 /-189 /-300]$} \\
& & & & & \\
SFSF & 1 & {$[-157 /-42 /-158 /-40]$} & CCFS & 1 & {$[-212 /-105 / 249 /-217]$} \\
& 2 & {$[-23 /-280 /-25 /-281]$} & & 2 & {$[-163 / 128 /-9 / 272]$} \\
& & & & & {$[-74 / 260 /-74 / 260]$} \\
SSSS & 1 & {$[-29 /-149 /-26 /-147]$} & CCCC & 1 & {$[-79 /-304 /-79 /-304]$} \\
& 2 & {$[-132 /-318 /-132 /-320]$} & & 2 & \\
\hline \hline
\end{tabular}

Table 1: Optimum solutions for composite laminated plates with $[0 / 0 / 0]_{\mathrm{S}}$ stacking sequence and $h / \bar{a}=0.01$ (Volt.) 


\begin{tabular}{cccccc}
\hline \hline BCs & $\bar{a} / \bar{b}$ & {$\left[\mathrm{~V}_{1} / \mathrm{V}_{2} / \mathrm{V}_{3} / \mathrm{V}_{4}\right]_{\mathrm{Opt}}$} & $\mathrm{BCs}$ & $\bar{a} / \bar{b}$ & {$\left[\mathrm{~V}_{1} / \mathrm{V}_{2} / \mathrm{V}_{3} / \mathrm{V}_{4}\right]_{\mathrm{Opt}}$} \\
\hline $\mathrm{CFFF}$ & 1 & {$[-158 /-52 /-159 /-50]$} & $\mathrm{CSCS}$ & 1 & {$[-155 /-22 /-155 /-22]$} \\
& & & & & \\
$\mathrm{SFSF}$ & 1 & {$[-157 /-40 /-153 /-41]$} & $\mathrm{CCFS}$ & 1 & {$[-212 /-105 / 249 /-217]$} \\
& & & & & \\
$\mathrm{SSSS}$ & 1 & {$[-29 /-147 /-29 /-147]$} & $\mathrm{CCCC}$ & 1 & {$[-74 / 260 /-72 / 264]$} \\
\hline \hline
\end{tabular}

Table 2: Optimum solutions for composite laminated plates with $[45 /-45 / 45]_{\mathrm{S}}$ stacking sequence and $h / \bar{a}=0.01$ (Volt.)

\begin{tabular}{cccccc}
\hline \hline BCs & $\bar{a} / \bar{b}$ & {$\left[\mathrm{~V}_{1} / \mathrm{V}_{2} / \mathrm{V}_{3} / \mathrm{V}_{4}\right]_{\mathrm{Opt}}$} & $\mathrm{BCs}$ & $\bar{a} / \bar{b}$ & {$\left[\mathrm{~V}_{1} / \mathrm{V}_{2} / \mathrm{V}_{3} / \mathrm{V}_{4}\right]_{\mathrm{Opt}}$} \\
\hline $\mathrm{CFFF}$ & 1 & {$[-269 /-223 /-265 /-227]$} & $\mathrm{CSCS}$ & 1 & {$[-163 / 242 /-163 / 241]$} \\
$\mathrm{SFSF}$ & 1 & {$[-245 / 334 /-245 / 334]$} & $\mathrm{CCFS}$ & 1 & {$[-363 /-255 / 149 /-178]$} \\
$\mathrm{SSSS}$ & 1 & {$[-39 /-349 /-35 /-348]$} & $\mathrm{CCCC}$ & 1 & {$[-219 / 317 /-219 / 317]$} \\
\hline \hline
\end{tabular}

Table 3: Optimum solutions for composite laminated plates with $[0 / 0 / 0]_{\mathrm{S}}$ stacking sequence and $h / \bar{a}=0.005$ (Volt.)

\begin{tabular}{cccccc}
\hline \hline BCs & $\bar{a} / \bar{b}$ & {$\left[\mathrm{~V}_{1} / \mathrm{V}_{2} / \mathrm{V}_{3} / \mathrm{V}_{4}\right]_{\mathrm{Opt}}$} & $\mathrm{BCs}$ & $\bar{a} / \bar{b}$ & {$\left[\mathrm{~V}_{1} / \mathrm{V}_{2} / \mathrm{V}_{3} / \mathrm{V}_{4}\right]_{\mathrm{Opt}}$} \\
\hline $\mathrm{CFFF}$ & 1 & {$[-159 /-53 /-156 /-55]$} & $\mathrm{CSCS}$ & 1 & {$[-157 /-21 /-154 /-22]$} \\
$\mathrm{SFSF}$ & 1 & {$[-157 /-40 /-157 /-40]$} & $\mathrm{CCFS}$ & 1 & {$[-212 /-105 / 249 /-217]$} \\
$\mathrm{SSSS}$ & 1 & {$[-30 /-147 /-29 /-147]$} & $\mathrm{CCCC}$ & 1 & {$[-74 / 262 /-71 / 266]$} \\
\hline \hline
\end{tabular}

Table 4: Optimum solutions for laminated plates with $[45 /-45 / 45 /-45 / 45]_{\mathrm{S}}$ stacking sequence and $h / \bar{a}=0.01$ (Volt.)

\section{CONCLUSIONS}

In this study, the voltages of patches optimization were carried out for minimizing the power consumption and maximum vertical displacement of symmetrically smart laminated composite plates by the EABC algorithm. The performance of the E-ABC was compared with the PSO algorithm and demonstrated the good efficiency of the E-ABC algorithm. As seen from the results, the E-ABC algorithm was successful in minimizing the power consumption and maximum vertical displacement of symmetrically composite plates using piezoelectric patches. In addition, the optimum voltages of patches were substantially influenced for edge conditions, plate length/width ratios and $\bar{a} / \bar{b}$ ratios. As seen, the optimum voltages of patches were not substantially influenced and approach a limiting value by changing fiber orientations and number of layers with the same plate thickness/length ratios. 


\section{References}

Chandrashekhara, K., Agarwal, A.N., (1993). Active vibration control of laminated composite plates using piezoelectric devices: a finite element approach, Journal of Intelligent Material Systems and Structures 4(4): 496-508.

Chen, J., Dawe, D.J., (1996). Linear transient analysis of rectangular laminated plates by a finite strip-mode superposition method, Composite Structures 35(2): 213-228.

Correia, V.M.F., Gomes, M.A.A., Suleman, A., Soares, C.M.M., Soares, C.A.M., (2000). Modelling and design of adaptive composite structures, Comput Methods Appl Mech Engrg 185: 325-346.

Devasia, S., Meressi, T., Brad Paden B., Eduardo Bayo, E., (1993). Piezoelectric actuator design for vibration suppression: placement and sizing, Journal of Guidance, Control, and Dynamics 16 (5): 859-864.

Elshafei, M.A., (1996). Smart Composite Plate Shape Control using Piezoelectric Materials, PhD Dissertation, U.S. Naval Postgraduate School, CA.

Ghashochi Bargh, H., Sadr, M.H., (2012). Stacking sequence optimization of composite plates for maximum fundamental frequency using particle swarm optimization algorithm, Meccanica 47(3): 719-730.

Ghashochi-Bargh, H., Sadr, M.H., (2013). PSO algorithm for fundamental frequency optimization of fiber metal laminated panels, Structural Engineering and Mechanics 47(5): 713-727.

Halim, D., Moheimani, S.O.R., (2003). An optimization approach to optimal placement of collocated piezoelectric actuators and sensors on a thin plate, Mechatronics 13(1): 27-47.

Han, J.H., Lee, I., (1999). Optimal placement of piezoelectric sensors and actuators for vibration control of a composite plate using genetic algorithms, Smart Materials and Structures 8(2): 257-267.

Jones. R.M., (1975). Mechanics of composite materials, Washington DC:Scripta.

Julai, S., Tokhi, M.O., (2010). Vibration suppression of flexible plate structures using swarm and genetic optimization techniques, Journal of Low Frequency Noise, Vibration and Active Control 29(4): 293-318.

Kang, Z., Tong, L., (2008). Topology optimization-based distribution design of actuation voltage in static shape control of plates, Computers \& Structures 86(19): 1885-1893.

Kapuria, S., Yasin, M.Y., (2013). Active vibration control of smart plates using directional actuation and sensing capability of piezoelectric composites, Acta Mechanica, 224(6): 1185-1199.

Karaboga, D., (2005). An idea based on honey bee swarm for numerical optimization, Technical report., Computer Engineering Department., Engineering Faculty., Erciyes University.

Koconis, D.B., Kollar, L.P., Springer, G.S., (1994). Shape control of composite plates and shells with embedded actuators. 2. desired shape Specified, Journal of Composite Materials 28(5): 459-482.

Li, G., Niu, P., Xiao, X., (2012). Development and investigation of efficient artificial bee colony algorithm for numerical function optimization, Applied soft computing 12(1): 320-332.

Maleki, S., Tahani, M., Andakhshideh, A., (2012). Transient response of laminated plates with arbitrary laminations and boundary conditions under general dynamic loadings, Archive of Applied Mechanics 82(5): 615-630.

Mezura-Montes, E., Velez-Koeppel, R.E., (2010, July). Elitist artificial bee colony for constrained real-parameter optimization, In: Evolutionary Computation (CEC)., 2010 IEEE Congress on; 1-8.

Montazeri, A., Poshtan, J., Yousefi-Koma, A., (2008). The use of 'particle swarm to optimize the control system in a PZT laminated plate, Smart Materials and Structures 17(4): 045027 (1-7). 
Onoda, J., and Hanawa, Y., (1993). Actuator placement optimization by genetic and improved simulated annealing algorithms, AIAA Journal 31(6): 1167-1169.

Qiu, Z.C., Zhang, X.M., Wu, H.X., Zhang, H.H., (2007). Optimal placement and active vibration control for piezoelectric smart flexible cantilever plate, Journal of Sound and Vibration 301(3): 521-543.

Rojas, J.E., Viana, F.A.C., Rade, D.A., (2004). Identification of external loads in mechanical systems through heuristicbased optimization methods and dynamic responses, Latin American Journal of Solids and Structures 1(3): 297-318.

Sadr, M.H., Ghashochi Bargh, H., (2012). Optimization of laminated composite plates for maximum fundamental frequency using Elitist-Genetic algorithm and finite strip method, J Glob Optim. 54: 707-728.

Sun, B., Huang, D., (2000). Analytical vibration suppression analysis of composite beams with piezoelectric laminae, Smart materials and structures 9(6): 751-760.

Sun, D., Tong, L., (2003). Optimum control voltage design for constrained static shape control of piezoelectric structures, AIAA Journal, 41(12): 2444-2450.

Sun, D.C., Tong, L.Y., (2005). Design optimization of piezoelectric actuator patterns for static shape control of smart plates, Smart Materials and Structures 14(6) 1352-1362.

Varadan, V.V., Kim, J., Varadan, V.K., (1997). Optimal placement of piezoelectric actuators for active noise control, AIAA Journal 35(3): 526-533.

Vinson, J.R., Sierakowski, R.L., (1986). The behavior of structures composed of composite materials, Dordrecht: Martinus Nijhoff. 